\title{
Geometric frustration in small colloidal clusters
}

\author{
Alex Malins ${ }^{1}$, Stephen Williams ${ }^{2}$, Jens Eggers ${ }^{3}$, Hajime \\ Tanaka $^{4}$ and C. Patrick Royall ${ }^{1}$ \\ ${ }^{1}$ School of Chemistry, University of Bristol, Bristol, BS8 1TS, UK \\ ${ }^{2}$ Research School of Chemistry, The Australian National University, Canberra, ACT \\ 0200, Australia \\ ${ }^{3}$ School of Mathematics University of Bristol University Walk Bristol BS8 1TW, UK \\ ${ }^{4}$ Institute of Industrial Science, University of Tokyo, 4-6-1 Komaba, Meguro-ku, \\ Tokyo 153-8505, Japan \\ E-mail: paddy.royall@bristol.ac.uk \\ Received May 20th, 2009
}

\begin{abstract}
We study the structure of clusters in a model colloidal system with competing interactions using Brownian dynamics simulations. A short-ranged attraction drives clustering, while a weak, long-ranged repulsion is used to model electrostatic charging in experimental systems. The former is treated with a shortranged Morse attractive interaction, the latter with a repulsive Yukawa interaction. We consider the yield of clusters of specific structure as a function of the strength of the interactions, for clusters with $m=3,4,5,6,7,10$ and 13 colloids. At sufficient strengths of the attractive interaction (around $10 k_{B} T$ ), the average bond lifetime approaches the simulation timescale and the system becomes nonergodic. For small clusters $m \leqslant 5$ where geometric frustration is not relevant, despite nonergodicity, for sufficient strengths of the attractive interaction the yield of clusters which maximise the number of bonds approaches $100 \%$. However for $m=7$ and higher, in the nonergodic regime we find a lower yield of these structures where we argue geometric frustration plays a significant role. $m=6$ is a special case, where two structures, of octahedral and $C_{2 v}$ symmetry compete, with the latter being favoured by entropic contributions in the ergodic regime and by kinetic trapping in the nonergodic regime. We believe that our results should be valid as far as the one-component description of the interaction potential is valid. A system with competing electrostatic repulsions and van der Waals attractions may be such an example. However, in some cases, the one-component description of the interaction potential may not be appropriate.
\end{abstract}

PACS numbers: 82.70.Dd; 82.70.Gg; 64.75.+g; 64.60.My

\section{Introduction}

Clusters are a distinct state of matter which exhibit different structural ordering relative to bulk materials [1]. Of particular relevance to, for example, many biological systems such as viruses, is their tendency to exhibit five-fold symmetry such as icosahedra and decahedra [2]. Recently there has been a surge of interest in clusters formed in colloidal 
systems [3, 4, 5, 6, 7, 8, 9, 10, 11, 14, 15, 18, which is expected to lead to the development of 'colloidal molecules' [4, 19, 10, 20, 15]. These may in turn provide novel functionalised materials [4, 19, 10, 11, 20, 15].

Part of the attraction of studying colloidal dispersions is that, although in principle they are rather complex multicomponent systems, the spatial and dynamic asymmetry between the colloidal particles $(10 \mathrm{~nm}-1 \mu \mathrm{m})$ and smaller molecular and ionic species has led to schemes where the smaller components are formally integrated out [21]. This leads to a one-component picture, where only the effective colloid-colloid interactions need be considered. This is usually a good approximation, however, there are a few exceptions. To describe the effective interactions between charged colloidal particles, the screening of the counter-ions is treated on a linear response level resulting in the well-known screened Coulomb pair potential proposed by Derjaguin, Landau, Verwey and Overbeek (DLVO). In general, however, due to non-linear counter-ion screening, the effective interactions involve many-body contributions [22], which violates the additivity of the potential. Furthermore, in strongly driven systems hydrodynamic interactions (velocity fields) modify the electrostatic interaction significantly [23, 24]. Here we assume the onecomponent description.

The colloid behaviour in the original complex system may then be faithfully reproduced by appealing to liquid state theory [25] and computer simulation [26]. Since the shape of the particles is typically spherical, and the effective colloid-colloid interactions may be tuned, it is often possible to use models of simple liquids to accurately describe colloidal dispersions. For example this approach has made it possible to reproduce Bernal spirals seen experimentally [6, 27] in a system with competing shortranged attractions and long-ranged repulsions, leading to the idea that colloidal gels can be stablised by repulsive interactions [28, 29, 30]. In addition to their own fascinating behaviour such as novel diffusion [15] colloidal clusters are also predicted from theory and simulation to exhibit hierarchical ordering such as lamellae [31], cluster crystals [32] and may also undergo dynamical arrest to form cluster glasses [28, 29, 33].

Since colloids may be directly imaged at the single-particle level, one may consider local intra-cluster structure, along with cluster-cluster correlations, a level of detail seldom accessible to atomic and molecular systems, except in the low-temperature regime [34]. Meanwhile, the behaviour of colloidal clusters, for example the global energy minimum structure, should exhibit similarities to that of clusters of Noble gas atoms as both colloids and Noble gases can be well described by spherically symmetric attractive interactions [35, 36]. Some of us recently compared direct imaging of colloidal clusters with expectations from theory [37]. In the experiments, a significant deviation from expectations was found, in particular a maximum yield of only around $10 \%$ in structures expected to minimise the potential energy for small $4 \leqslant m \leqslant 6$ clusters. This is surprising, as at these small sizes, there is little geometrical frustration that might inhibit access to the ground state, as would occur for larger clusters.

It is the purpose of this work to establish those cluster structures we expect in the case of model colloidal systems by applying tried and tested model interactions. We 
shall consider weak electrostatic repulsions and a short-ranged attraction for similar parameters to the experimental system. Typically, the former stems from electrostatic charge, the latter from the addition of non-adsorbing polymer or van der Waals attractions, and drives clustering. Rather than seeking minimum energy states [35, 36], instead we shall try to mimic experimental approaches, as a way to predict what sort of yields of desired clusters we find as a function of interaction strength. Experiments on colloidal systems typically start from a randomised initial state. Unlike molecular systems, the effective temperature is typically constant: temperature itself is not usually varied and the effective colloid-colloid interactions in a given sample are taken to be fixed, as they depend upon sample composition. In other words the system undergoes an 'instantaneous quench' from an initial, randomised state. We follow this protocol, although we note recent theoretical and simulation work highlighting the role of microscopic reversibility in optimising yields in self-assembling systems [38, 39, 40] which is beginning to be exploited in nanoscience [41. For the larger cluster sizes considered, we also investigate the role of electrostatic repulsions in cluster elongation, as suggested theoretically [42] and noted experimentally [5, 6, 7].

Our approach is as follows. We shall consider clusters of $m=3,4,5,6,7,10,13$ particles and study the structures formed, with particular reference to the minimum energy states [35, 36], as a function of the attractive interaction strength (which mimics the addition of polymer in experimental systems). Various strengths of the repulsive interaction (which models the electrostatic charge in experimental systems) are also considered. For high strengths of the attractive interaction (low effective temperature), one expects the majority of clusters to reside in their minimum energy state. However, average bond lifetimes can exceed the simulation run time (which is comparable to experimental timescales) for sufficiently strong interactions. The system is thus nonergodic on these timescales, and kinetic trapping may become important. For larger clusters, long-ranged repulsions may lead to elongation [5, 6, 7, 27, 36, 42]. We investigate this effect for $m=10,13$. Our main result is that for small clusters $(m \leqslant 5)$ the lack of geometric frustration enables the minimum energy state to be accessed, while for larger clusters kinetic trapping is important.

This paper is organised as follows: section 2 introduces the model interactions, our simulation methodology is presented in section 3, followed by results (section 4) and a discussion in section 5 in which we place our findings in the context of recent work. We conclude our findings in section 6 .

\section{Model}

The seminal theory of colloid-polymer mixtures is that of Asakura and Oosawa [43]. This AO model ascribes an effective pair interaction between two colloidal hard spheres 
in a solution of ideal polymers which is plotted in figure 1 and reads,

$$
\beta u_{A O}(r)= \begin{cases}\frac{\pi\left(2 R_{G}\right)^{3} z_{P R}}{6} \frac{(1+q)^{3}}{q^{3}} & \text { for } r \leq \sigma, \\ \times\left\{1-\frac{3 r}{2(1+q) \sigma}+\frac{r^{3}}{2(1+q)^{3} \sigma^{3}}\right\} & \text { for } \sigma<r \leq \sigma+\left(2 R_{G}\right), \\ 0 & \text { for } r>\sigma+\left(2 R_{G}\right),\end{cases}
$$

where $\beta=1 / k_{B} T, r$ is the centre to centre separation of the two colloids and the polymer fugacity $z_{P R}$ is equal to the number density $\rho_{P R}$ of ideal polymers in a reservoir at the same chemical potential as the colloid-polymer mixture. Thus within the AO model the effective temperature is inversely proportional to the polymer reservoir concentration. The polymer-colloid size ratio $q=2 R_{G} / \sigma$ where $R_{G}$ is taken as the polymer radius of gyration, and $\sigma$ is the colloid diameter. For small polymer-colloid size ratios, the AO model has been found to give good agreement with direct experimental measurement [44].

The discontinuous nature of the $\mathrm{AO}$ interaction at contact complicates its use in Brownian dynamics simulations. We therefore use the continuous Morse potential, which, for short interaction ranges, is very similar to the Asakura-Oosawa potential (figure 1) [45]. The Morse potential reads

$$
\beta u_{M}(r)=\beta \varepsilon_{M} e^{\rho_{0}(\sigma-r)}\left(e^{\rho_{0}(\sigma-r)}-2\right),
$$

where $\rho_{0}$ is a range parameter and $\beta \varepsilon_{M}$ is the potential well depth. The global energy minimum structures for clusters interacting via the Morse interaction are known [35], and for small clusters $m<8$, the structure of the global energy minimum is not sensitive to the range of the interaction. With the exception of $m=6$ (see below), small ground state clusters should be the same for an AO colloid-polymer system as those tabulated for the Morse interaction. The experimental system [37] used a polymer-colloid size ratio of $q=0.22$ which maps to a Morse range parameter $\rho_{0}=33.06$ for a well depth $\beta \varepsilon_{M}=5.0$ according to the extended law of corresponding states [46].

Repulsions in colloidal systems typically stem from the electrostatic charge on the colloidal particles. Under many conditions, where the charging is quite weak, as is the case here, this leads to a screened Coulomb, or Yukawa form with a hard core that accounts for the physical size of the colloidal particles:

$$
\beta u_{Y}(r)= \begin{cases}\infty & \text { for } r<\sigma, \\ \beta \varepsilon_{Y} \frac{\exp (-\kappa(r-\sigma))}{r / \sigma} & \text { for } r \geq \sigma,\end{cases}
$$

where $\kappa$ is the inverse Debye screening length. The contact potential is given by

$$
\beta \varepsilon_{Y}=\frac{Z^{2}}{(1+\kappa \sigma / 2)^{2}} \frac{l_{B}}{\sigma},
$$



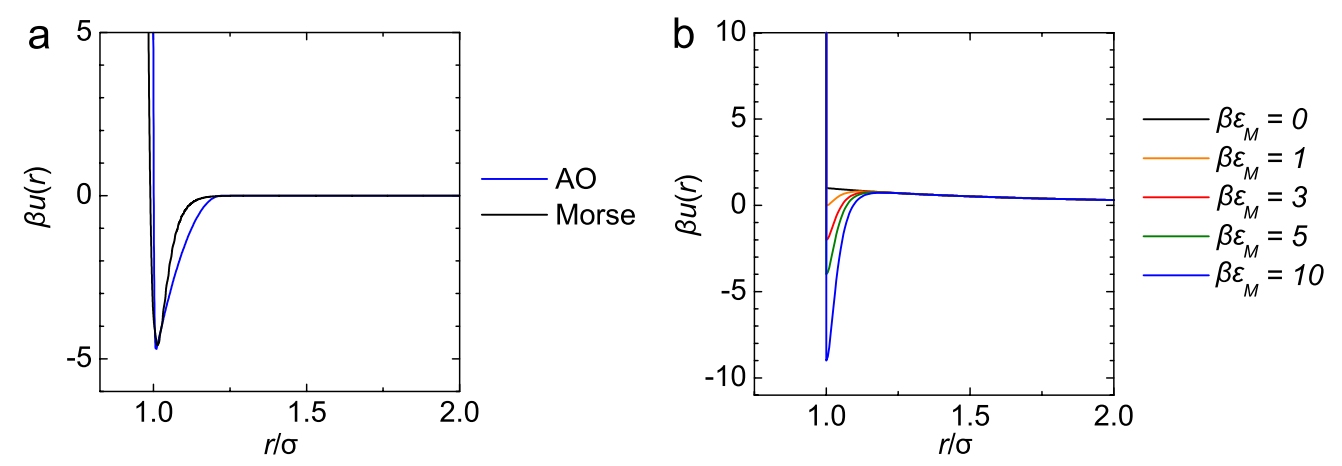

Figure 1. (color online) (a) The Morse potential [eq. (2)] matched to the AsakuraOosawa (AO) potential [eq. (1D] relevant to experimental systems. (b) The competing interactions used in this work, $\beta u_{M}+\beta u_{Y}$ for various values of the Morse potential well depth $\beta \varepsilon_{M}$ as shown in the legend. The case of $\beta \varepsilon_{M}=0$ is the pure Yukawa interaction [eq. (3)] contact potential of $\beta \varepsilon_{Y}=k T$ and inverse Debye screening length $\kappa \sigma=0.5$.

where $Z$ is the colloid charge and $l_{B}$ is the Bjerrum length. To model the experimental system, we therefore fix the Debye length to an experimentally relevant value $\kappa \sigma=0.5$, and consider different values of the contact potential $\beta \varepsilon_{Y}$. In the experimental system we seek to model, van der Waals attractions are largely absent, due to solvent-colloid refractive index matching, the attractions are driven by the addition of non-absorbing polymer.

We investigate the structure of these simulated colloidal clusters, with reference to the Morse clusters [35], using a new method we have developed, which we term the topological cluster classification (TCC) [45, 47]. Although we consider electrostatic repulsions, we expect little effect on the ground state for small $m<10$ clusters [36].

\section{Simulation and analysis}

We use a standard Brownian dynamics simulation scheme [48]. The scheme generates a discrete coordinate trajectory $\mathbf{r}_{i}$ as follows

$$
\mathbf{r}_{i}(t+\delta t)=\mathbf{r}_{i}(t)+\frac{D}{k_{B} T} \sum_{j=1, j \neq i}^{N} \mathbf{F}_{i j}(t) \delta t+\delta \mathbf{r}_{i}^{G},
$$

where $\delta t$ is the simulation time step, $D$ is the diffusion constant and $\mathbf{F}_{i j}$ is the pairwise interaction. The colloids respond to the direct interactions $\mathbf{F}_{i j}$ and the solvent-induced thermal fluctuations $\delta \mathbf{r}_{i}^{G}$ are treated as a Gaussian noise with the variance given by the fluctuation-dissipation theorem.

In the simulations $m$ particles are initialised randomly at volume fraction $\phi=$ $m\left(\pi \sigma^{3} / 6\right) / l^{3}=0.0029$ in a cubic box of side $l$. Periodic boundary conditions for the box are implemented. We study the evolution of one cluster per simulation, and consider the case where all particle are part of the cluster. The inter-particle interaction is 
the Morse potential [eq. (2)] with range parameter $\rho_{0}=33.06 \sigma^{-1}$ which is truncated and shifted for $r>3 \sigma$, where the Morse potential is typically less than $10^{-27}$. The electrostatic interactions are treated by adding a Yukawa repulsion term [eq. (3) ] for different values of $\beta \varepsilon_{Y}$ as specified and this is also truncated and shifted for $r>3 \sigma$. This rather short value enables the particles to form clusters more easily starting from the initial randomised state. While the Yukawa repulsion has not fully decayed at $r=3 \sigma$, the largest separation of two particle centres we are interested in is set by the size of the clusters. The largest separations for which we consider the effects of the Yukawa repulsion are the $m=13$ clusters, where the maximum separation is less than $3 \sigma$. Since the Morse interaction has no hard core, we remove the hard core component of eq. (3). Each state point is sampled with between four and twelve statistically independent simulation runs.

The time-step is $\delta t=0.03$ simulation time units and all runs are equilibrated for $10^{9}$ steps and run for further $10^{9}$ steps. The rather long simulation runs were required to be sure that, in the case of Yukawa repulsions, that the particles had ample time to interact with one another, and cluster. We define the Brownian time as the time taken for a colloid to diffuse its own radius:

$$
\tau_{B}=\frac{\sigma^{2}}{4 D}
$$

In the simulations, $\tau_{B} \approx 2474$ time units, while in the experiments $\tau_{B} \sim 10 \mathrm{~s}$ [37]. The simulation runs therefore correspond to a total of around 68 hours, a timescale certainly comparable to experimental work.

We identify two particles as bonded if the separation of the particle centres is less than $1.25 \sigma$, which is close to the attractive range of a strict AO potential [eq. (11)]. Having identified the bond network, we use the Topological Cluster Classification (TCC) to determine the nature of the cluster [47]. This analysis identifies all the shortest path three, four and five membered rings in the bond network. We use the TCC to find clusters which are global energy minima of the Morse potential. We follow Doye et.al. [35] and term these clusters 3A, 4A, 5A, 6A, 7A, 10B, and 13B for $m=3,4,5,6,7,10,13$. For $m \leqslant 7$ there is one global minimum for the Morse potential. At higher $m$ there are multiple minima corresponding to different values of the range parameter $\rho_{0}$. $10 \mathrm{~B}$ and $13 \mathrm{~B}$ correspond to a short ranged Morse potential. We assume that these are the relevant global minima for $\rho_{0}=33.06$. In addition, in the case of $m=13$ clusters we identify the FCC and HCP thirteen particle structures in terms of a central particle and its twelve nearest neighbours. For more details see [47].

\section{Results}

We shall consider each size in turn, before drawing together our results. We use two control parameters, the well depth of the attractive Morse interaction $\beta \varepsilon_{M}$ [eq. (2)] and the contact potential of the Yukawa repulsion $\beta \varepsilon_{Y}$ [eq. (3)]. Increasing $\beta \varepsilon_{M}$ thus promotes clustering, while $\beta \varepsilon_{Y}$ suppresses clustering. In experiments on colloids, the 

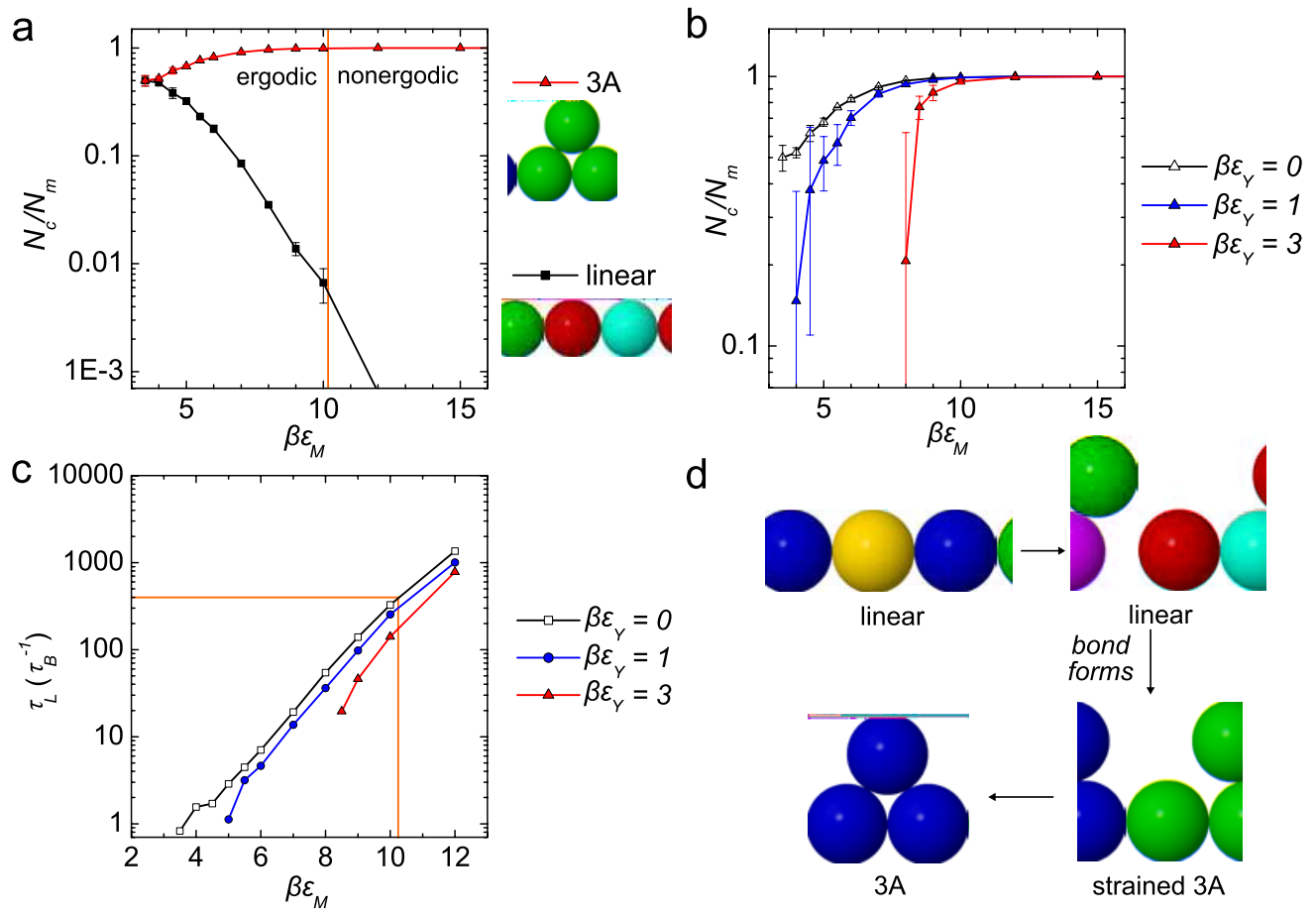

d

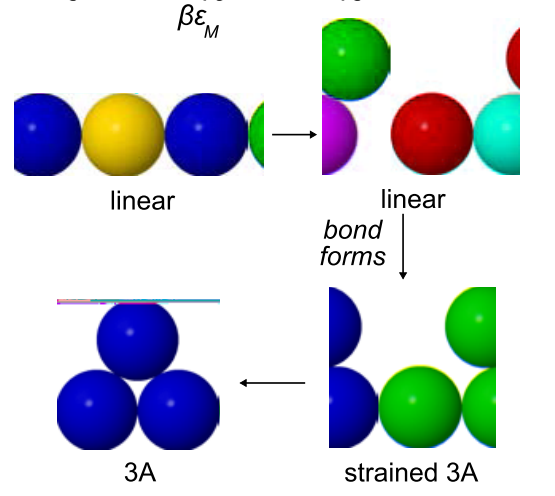

Figure 2. (color online) (a) Cluster populations for the $m=3$ system as a function of the well depth of the attractive interaction $\beta \varepsilon_{M}$ with $\beta \varepsilon_{Y}=0$. Here we define cluster population by the ratio $N_{c} / N_{m}$ where $N_{c}$ and $N_{m}$ are the total number of specific clusters and the total number of $m$ membered clusters identified respectively in the simulation after equilibration. We see a clear trend towards the $3 \mathrm{~A}$ triangle as the minimum energy ground state structure. The orange line corresponds to $\tau_{L}=400 \tau_{B}$, our criterion for ergodicity breaking on the simulation timescale [see (c)]. (b) population of clusters in the minimum energy state (3A triangle) for different strengths of the Yukawa interaction $\beta \varepsilon_{Y}$. The principal effect of the Yukawa repulsion is to shift the curves to require higher values of $\beta \varepsilon_{M}$ to achieve the same population of clusters in the ground state. (c) the average bond lifetime $\tau_{L}$ as a function of $\beta \varepsilon_{M}$ for various $\beta \varepsilon_{Y}$ as indicated. The orange lines correspond to $\tau_{L}=400 \tau_{B}$. (d) the linear $\rightarrow 3 \mathrm{~A}$ transition can be accomplished by rotating one end particle around the central particle. This process involves no energetic penalty for $\beta \varepsilon_{Y}=0$.

electrostatic charge is usually not systematically varied. Therefore we consider specific values of $\beta \varepsilon_{Y}$ and plot the response of the system to $\beta \varepsilon_{M}$. Small clusters with $m \leqslant 5$ are able to reach the minimum energy ground state structures (which maximise the number of bonds), while for larger clusters, geometric frustration leads to kinetic trapping which severely limits access to the ground state at high values of the attractive interaction.

\subsection{Small clusters $m \leqslant 5$}

We begin our presentation of results by considering the $m=3$ system, as shown in figure 2(a). The main conclusion from these data, as with all $m \leq 5$, is that increasing the attractive interaction strength $\beta \varepsilon_{M}$ leads to a higher population in the minimum energy ground state, here the ' $3 \mathrm{~A}$ ' triangle with $D_{3 h}$ point group symmetry and three 
bonds. This occurs at the expense of higher energy clusters, which, for $m=3$ are 'linear' clusters with two bonds. In the case of $\beta \varepsilon_{Y}=0$, the potential energy for a short-range potential such as eq. (2) is approximately equal to the number of bonds. Since $3 \mathrm{~A}$ triangles have three bonds, and the linear clusters have only two, there is a strong thermodynamic driving force to the $3 \mathrm{~A}$ cluster as the attractions are increased, consistent with the behaviour seen in figure 2(a).

The effect of increasing the Yukawa repulsion is to slightly suppress the development of the 3A population [figure 2(b)], as expected in this system with competing interactions. In other words, the slight upwards shift in the potential $\beta u_{M}+\beta u_{Y}$ [figure 1(b)] due to the Yukawa contribution acts as a relatively small perturbation to the $m=3$ system. However, $\beta \varepsilon_{Y}=5$ substantially suppressed the colloidal aggregation and few three-membered clusters were formed. We hereafter consider $\beta \varepsilon_{Y}=0,1,3$ only.

The average bond lifetime $\tau_{L}$ is also shown in figure 2(c). Here we define bond lifetime as the time between a bond formation event (where the separation between two colloids falls below the bond length 1.25 ) a bond breakage event (where the separation between the same two colloids rises above the bond length). The bond lifetimes were widely distributed in all cases. We see that for $\beta \varepsilon_{M} \lesssim 10, \tau_{L}$ is very much less than the simulation time, so the system may be regarded as equilibrated. In this regime, $\tau_{L}$ exhibits an Arrhenius-like behaviour, as expected for an equilibrated system. At higher values of the interaction strength, the average bond lifetime approaches, and then exceeds the simulation run time, so the system is unable to equilibrate, on the simulation (and experimental [37]) timescale. Thus the system is regarded as nonergodic on these timescales, in that it cannot explore all its configurations. We take a average bond lifetime $\tau_{l}=400 \tau_{B}$ as a crossover between ergodic and nonergodic. This ergodic - nonergodic transition is indicated in figure 2(a). However, although the system is nonergodic, the absence of any geometric frustration enables the minimum energy ground state to be reached. The absence of geometric frustration is understood as follows [figure 2(d)]: for $m=3$, if $\beta \varepsilon_{Y}=0$ there is no energy barrier in the linear $\rightarrow 3 \mathrm{~A}$ transition, because there is no angular dependence in the interaction. In other words, a steepest descent quench for a three-membered cluster yields the $3 \mathrm{~A}$ triangle.

Turning to the case for $m=4$, the situation is somewhat more complex [figure 3(a)] Rather than two states, there are four: 4A tetrahedra (the ground state with 6 bonds and $T_{h}$ point group symmetry) diamonds ( 5 bonds), triangle-lines and squares ( 4 bonds) and linear (3 bonds). Squares are distinct from diamonds in that there are no diagonal bonds. However, like the $m=3$ case, increasing $\beta \varepsilon_{M}$ we find a peak in the population of linear clusters, triangle-lines and diamonds respectively. Each higher energy state has a distribution which becomes progressively less favoured at higher values of $\beta \varepsilon_{M}$, as the $4 \mathrm{~A}$ becomes the dominant structure, the yield of $4 \mathrm{~A}$ approaches unity for $\beta \varepsilon_{M}>10$. Squares have a rather low yield, much less than triangle-lines $(3 \mathrm{~A}+1)$, which have the same number of bonds. We return to the case of competing structures during our analysis of $m=6$.

The effect of increasing the Yukawa interaction is similar to the $m=3$ case: 

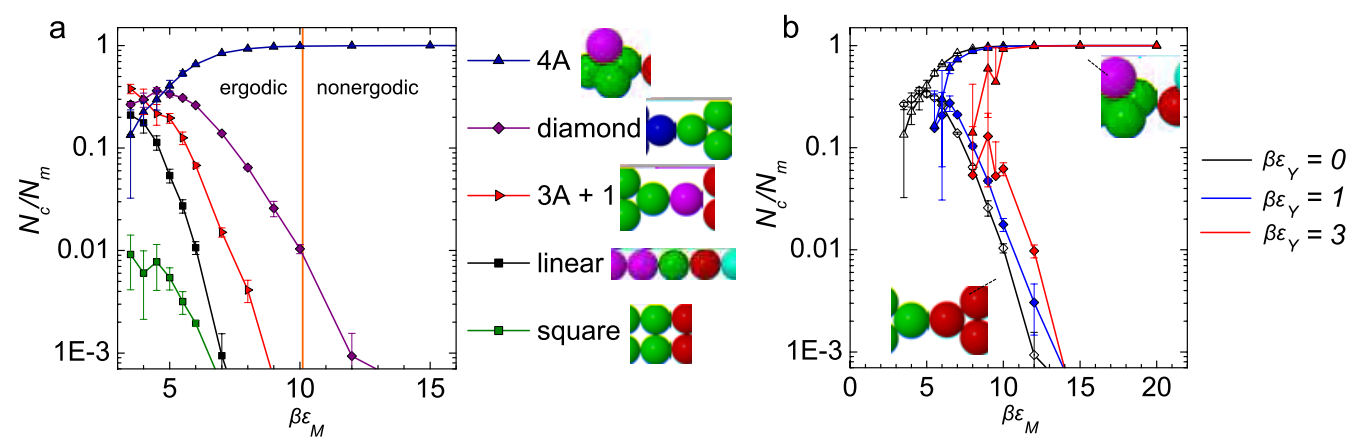

Figure 3. (color online) (a) Cluster populations for the $m=4$ system as a function of the well depth of the attractive interaction $\beta \varepsilon_{M}$ with $\beta \varepsilon_{Y}=0$. Here we see a clear trend towards the $4 \mathrm{~A}$ tetrahedron as the ground state structure. We identify three higher energy structures: diamonds ( 5 bonds), triangle-lines (or $3 \mathrm{~A}+1)(4$ bonds) and linear (3 bonds). The orange line corresponds to $\tau_{l}=400 \tau_{B}$. (b) population of clusters in the minimum energy ground state ( $4 \mathrm{~A}$ tetrahedron) and extended diamond structure for different strengths of the Yukawa repulsion $\beta \varepsilon_{Y}$. The principle effect of the Yukawa repulsion is to shift the curves to require higher interaction strengths to achieve the same degree of clusters in the ground state: the more elongated diamond structure does not appear more favoured for stronger Yukawa repulsions.

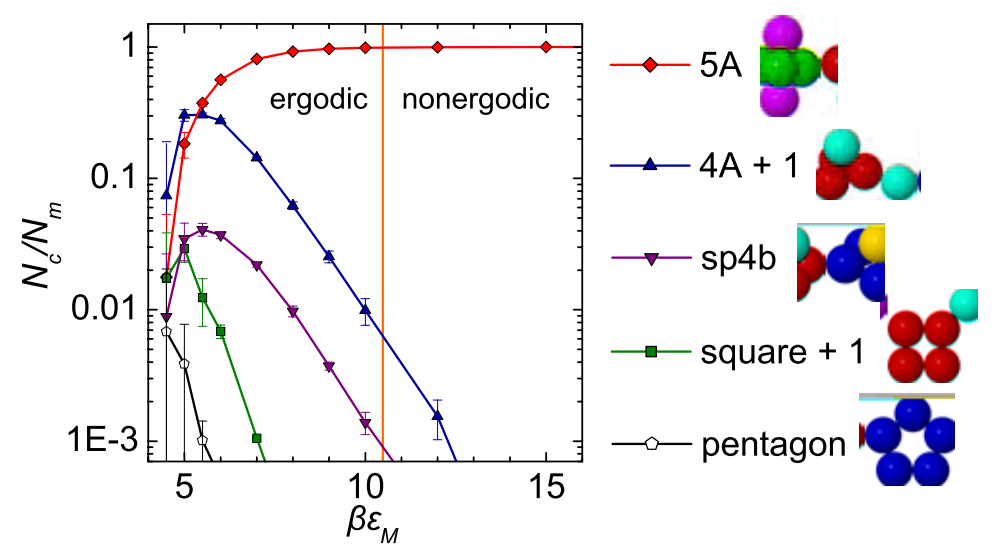

Figure 4. (color online) Cluster populations for the $m=5$ system as a function of the well depth of the attractive interaction $\beta \varepsilon_{M}$ with $\beta \varepsilon_{Y}=0$. The orange line corresponds to $\tau_{l}=400 \tau_{B}$. sp $4 \mathrm{~b}$ denotes a four-membered ring with one particle bonded to all four in the ring.

the development of the $4 \mathrm{~A}$ population is somewhat suppressed [figure 3(b)]. It has been suggested that the introduction of repulsions might be expected to promote more elongated structures [42]. For a given strength of the attractive interactions $\beta \varepsilon_{M}$, there is indeed a tendency towards a higher population of the elongated $4 \mathrm{D}$ diamond structure, however the overall trend is unaltered and we restrict our analysis to the $\beta \epsilon_{Y}=0$ case.

We now consider the $m=5$ system for $\beta \varepsilon_{Y}=0$ [figure 4(a)]. We find that for $\beta \varepsilon_{M} \gtrsim 8.0$, the overwhelming majority of clusters are in the ground state, $5 \mathrm{~A}$ (triangular 


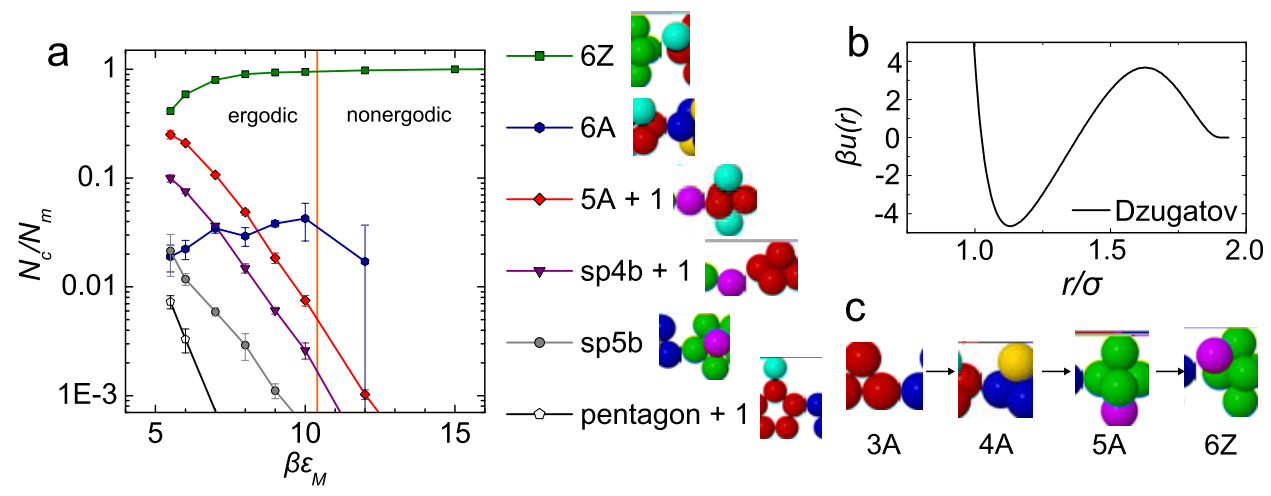

Figure 5. (color online) (a) Cluster populations for the $m=6$ system as a function of the well depth of the attractive interaction $\beta \varepsilon_{M}$ with $\beta \varepsilon_{Y}=0$. Here at strong interaction strength, rather than the Morse ground state $6 \mathrm{~A}$ octahedron, instead $6 \mathrm{Z}$ are found, the ground state for the Dzugutov potential, (b). The orange line corresponds to $\tau_{L}=400 \tau_{B}$. (c) The $3 \mathrm{~A} \rightarrow 4 \mathrm{~A} \rightarrow 5 \mathrm{~A} \rightarrow 6 \mathrm{Z}$ aggregation pathway with does not involve the breaking of any bonds and thus promotes the formation of $6 \mathrm{Z}$ over $6 \mathrm{~A}$ for high values of $\beta \varepsilon_{M}$ where the average bond lifetime $\tau_{l}$

is comparable to or greater than the simulation runtime.

bipyramid with 9 bonds and $D_{3 h}$ point group symmetry), in a similar way to the $m=3$ and $m=4$ cases. Defective triangular bipyramids $(4 \mathrm{~A}+1)$ form an excited state with 10 or 11 bonds. Clusters based around 4-membered rings with 5-8 bonds (sp4b and squares+1) are present in yields up to a few percent for relatively weak attractions $\left(\beta \varepsilon_{M} \sim 5\right)$. Five-membered rings (pentagons, five bonds) are found in small quantities, similar to the square in the case of $m=4$. The main result from considering these small clusters is that, although the system may become non-ergodic on the simulation timescale, the clusters can nevertheless access their global energy minima for sufficient interaction strengths $\left(\beta \varepsilon_{M} \gtrsim 8.0\right)$. In other words, access to the global minimum is not geometrically frustrated.

For $m=5$ we do not explicitly consider all possible structures. For example linear clusters may form at weaker interaction strengths. At $\beta \varepsilon_{M}=4.5$, for example, only around $10 \%$ of $m=5$ clusters are identified, however for most interaction strengths we consider, the vast majority of clusters are one of the five structures considered. A similar argument holds for $m=6$ and $m=7$.

\section{2. $m=6$ clusters: competing structures}

For $3 \leq m \leq 5$ the dominant structure at high interaction strength is the global energy minimum for the Morse interaction. However, in the case of $m=6$, we find this is not the case [figure 5(a)]. We see only a small population of the Morse global energy minimum, the $6 \mathrm{~A}$ octahedron ( $O_{h}$ point group symmetry) with another structure of $C_{2 v}$ point group symmetry appearing to dominate the system. This cluster is the ground 
state for the Dzugutov potential [plotted in figure 5(b)] [49], and we term it the 6Z. Why should this Dzugutov cluster be more popular than 6A octahedra? Both clusters have 12 bonds (near-neighours), and for a Morse potential with a relatively short range as we use here, the energy contribution from second-nearest neighbours is a factor of $5.625 \times 10^{-8}$ of the total energy for the $6 \mathrm{~A}$ (it is essentially zero for the $6 \mathrm{Z}$ ). In fact, when the Yukawa repulsions are added, $6 \mathrm{Z}$ becomes energetically favoured as it is more elongated. Let us however consider the case for neutral particles where $\beta \varepsilon_{Y}=0$.

The difference between the two clusters' ground state potential energies is slight, but there are two remaining contributions to the system's free energy to be considered. Firstly the contribution due to the vibrational modes of the clusters must be accounted for, and secondly, so must the volume of phase space which is accessed upon translating and rotating the clusters through the system volume. Assuming that the vibrational modes may be approximated as harmonic (valid at low temperatures), the vibrational contribution to the free energies can be computed using a standard normal mode analysis. We have done this and found the contribution due to the vibrational modes to be completely negligible. The contribution due to translation will be the same for both clusters, so need not be considered. This leaves the contribution due to rotating the clusters. For the rotation we must consider both the point group symmetry and the cluster's radius of gyration. Comparing the point group symmetries, we can see that there are 24 different ways to reorientate the $6 \mathrm{~A}$ cluster which are merely a permutation of indistinguishable particles, while for the $6 \mathrm{Z}$ there are only two ways. In computing the entropy we must count each permutation of indistinguishable particles only once. For this reason, in computing the entropy, we are able to rotate the $6 \mathrm{Z}$ cluster through a greater portion of the available phase space. This results in an increase in the $6 \mathrm{Z}$ cluster's population relative to the $6 \mathrm{~A}$ cluster's by a factor of 12 . One could think of the $6 \mathrm{Z}$ cluster's lower symmetry as a form of increased disorder. We also consider the radius of gyration: $R_{G}^{2}=\frac{1}{N} \sum_{i=1}^{6}\left(\mathbf{r}_{i}-\mathbf{r}_{c m}\right)^{2}$ where $\mathbf{r}_{i}$ are the particle coordinates and $\mathbf{r}_{c m}$ is the centre of mass. $R_{G}$ is larger by a factor of approximately 1.06 times for the $6 \mathrm{Z}$ cluster than it is for the $6 \mathrm{~A}$. So upon rotating the $6 \mathrm{Z}$ cluster its particles traverse a greater portion of the available phase space than is the case for the $6 \mathrm{~A}$ cluster. This increases the entropy of the $6 \mathrm{Z}$ cluster, relative to that of the $6 \mathrm{~A}$, further favouring it. It seems plausible that this could account for the relative differences in the populations, i.e., that the $6 \mathrm{Z}$ is thermodynamically favoured over the $6 \mathrm{~A}$ by a factor of 30 as shown in figure 5 (a).

As $\beta \epsilon_{M}$ becomes very large, we would ultimately expect a trend towards a $6 \mathrm{~A}$ dominated population due to the (small) difference in potential energy. However, on these simulation timescales, the average bond lifetime is too long to enable the transition to a $6 \mathrm{~A}$ dominated population, and, since $6 \mathrm{Z}$ can be the result of a $3 \mathrm{~A} \rightarrow 4 \mathrm{~A} \rightarrow 5 \mathrm{~A} \rightarrow 6 \mathrm{Z}$ aggregation sequence [Fig $5(\mathrm{c})]$, and the formation of $6 \mathrm{~A}$ requires bond breakage, at strong interaction strengths, $6 \mathrm{Z}$ dominates for kinetic reasons.

Like the smaller clusters, for $m=6$, we identify different structures which become significant at lower values of $\beta \varepsilon_{M}$. In decreasing stability, these are clusters with two 


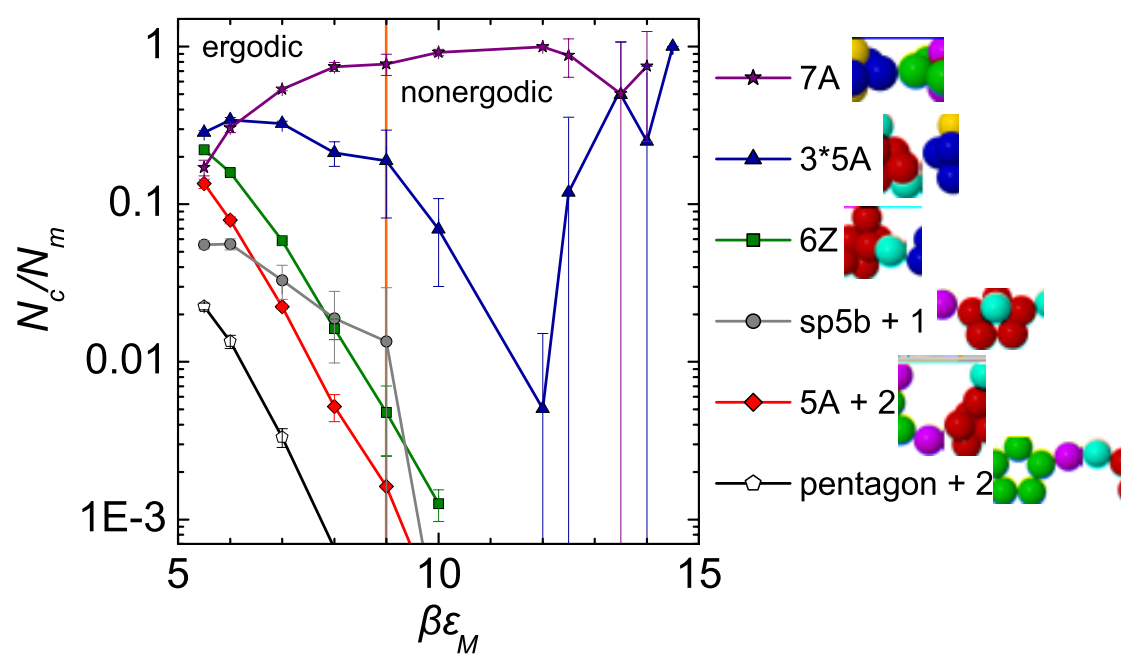

Figure 6. (color online) Cluster populations for the $m=7$ system as a function of the well depth of the attractive interaction $\beta \varepsilon_{M}$ with $\beta \varepsilon_{Y}=0$. Here the Morse ground state $7 A$ pentagonal bipyramid is readily formed at intermediate interaction strength. The orange line corresponds to $\tau_{l}=400 \tau_{B}$. sp5b denotes a five-membered ring with one particle bonded to all five in the ring. A cluster based on three overlapping $5 \mathrm{~A}$ triangular bipyramids, $3 \times 5 \mathrm{~A}$, shows a re-entrant behaviour, dominating the cluster population at high and low values of $\beta \varepsilon_{M}$.

tetrahedra [which we denote as $5 \mathrm{~A}+1$ in figure [5(a), with either 10 or 11 bonds], defective octahedra (denoted as sp4b+1, with between 9 and 11 bonds), defective pentagonal bipyramids (denoted as sp5b with 10 bonds) and clusters formed of a five-membered ring with one bound particle (pentagon +1 , between 6 and 9 bonds).

\subsection{Larger clusters: geometric frustration}

For the small clusters we have considered so far, the number of particles is apparently too small to form metastable states. However, for $m=7$ we see that the yield of the Morse global energy minimum 7A pentagonal bipyramid approaches unity for moderate strengths of $\beta \varepsilon_{M}$, but for $\beta \varepsilon_{M}>12$ not all simulations reach the 7A [figure 6(a)]. Once the $7 \mathrm{~A}$ is formed, the system remains in a $7 \mathrm{~A}$ state, but other metastable states have lifetimes longer than the simulation runs. The rise in $7 \mathrm{~A}$ population as a function of $\beta \varepsilon_{M}$ appears to slow around the ergodic-nonergodic transition (which we define as $\left.\tau_{L}=400 \tau_{B}\right)$.

In the nonergodic regime where bond breaking governs those structures which form, we expect an aggregation sequence similar to the $3 \mathrm{~A} \rightarrow 4 \mathrm{~A} \rightarrow 5 \mathrm{~A} \rightarrow 6 \mathrm{Z}$ sequence shown in figure 5(c). Stepwise aggregation of one particle onto a $6 \mathrm{Z}$ cluster would lead to a structure we term $3 \times 5 \mathrm{~A}$, as it may be decomposed into 3 overlapping $5 \mathrm{~A}$ triangular bipyramids, or, equivalently, a $6 \mathrm{Z}$ with an additional tetrahedron. This structure has 15 bonds. The $7 \mathrm{~A}$ has 15 bonds where the separation $\approx \sigma$, close to the minimum of 

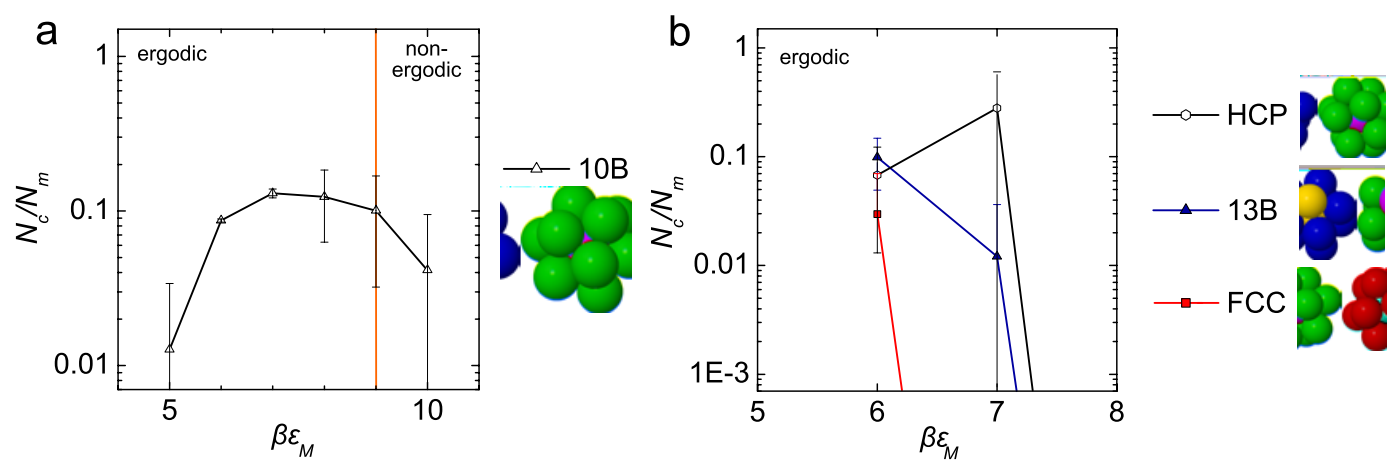

Figure 7. (color online) (a) Cluster populations for the $m=10$ (a) and $m=13$ (b) system as a function of the well depth of the attractive interaction $\beta \varepsilon_{M}$ with $\beta \varepsilon_{Y}=0$. The orange line in (a) corresponds to $\tau_{l}=400 \tau_{B}$

the Morse potential, while the distance between the top and bottom particle is around $1.02283 \sigma$, contributing $0.7193 \varepsilon_{M}$ to the energy. The $7 \mathrm{~A}$ is thus around $0.7193 \beta \varepsilon_{M}$ lower in energy than the $3 \times 5 \mathrm{~A}$ structure. In the nonergodic regime for $\beta \varepsilon_{M}>12$, we see that this $3 \times 5 \mathrm{~A}$ structure dominates the system, due to kinetic trapping. The behaviour of the 7A system in the nonergodic regime highlights the sampling limitations of our simulation approach. To map this regime more accurately, many more simulations are desirable than the 4 runs per state point we have been able to perform.

At weaker interaction strengths, like the smaller clusters, a variety of structures become popular, based on a diminishing number of bonds. Of these, sp $5 \mathrm{~b}+1$, a defective pentagonal bipyramid with 11-13 bonds shows a rather slow rate of decay upon increasing $\beta \varepsilon_{M}$. At the weakest interaction strength, $\beta \varepsilon_{M}=5.5$, the yield of $3 \times 5 \mathrm{~A}$ exceeds that of $7 \mathrm{~A}$. This 're-entrant' behaviour of $3 \times 5 \mathrm{~A}$ is apparently a consequence of the different number of bonds relative to 7A. Cluster with fewer bonds are promoted for weak interaction strengths, but in the nonergodic regime, kinetic trapping promotes $3 \times 5 \mathrm{~A}$.

At larger sizes again we consider the Morse global minima, which are 10B for 13B for $m=10,13$ clusters respectively. Once more the effects of frustration are clear. The $m=10$ system (figure 7) features a clear maximum yield of $10 \mathrm{~B}$ at $\beta \varepsilon_{M} \sim 7$, however the yield is only around $14 \%$. The rise in the yield of $10 \mathrm{~B}$ as a function of $\beta \varepsilon_{M}$ up to the ergodic-nonergodic crossover suggests that this rise in average bond lifetime is the primary mechanism by which a further rise in the $10 \mathrm{~B}$ yield is suppressed. In other words, kinetic trapping prevents access to $10 \mathrm{~B}$ at higher strengths of the attractive interaction.

For $m=13$, in addition to 13B, we also find crystal fragments. The maximum yield of $13 \mathrm{~B}$ is around $10 \%$. For $\beta \varepsilon_{M}=7.0, \mathrm{HCP}$ crystal fragments are in fact more popular than the 13B ground state. We find no icosahedra, these are the global energy minimum for longer ranged Morse interactions $\left(\rho_{0}<14.76\right)$ [35] than we use here, although for 

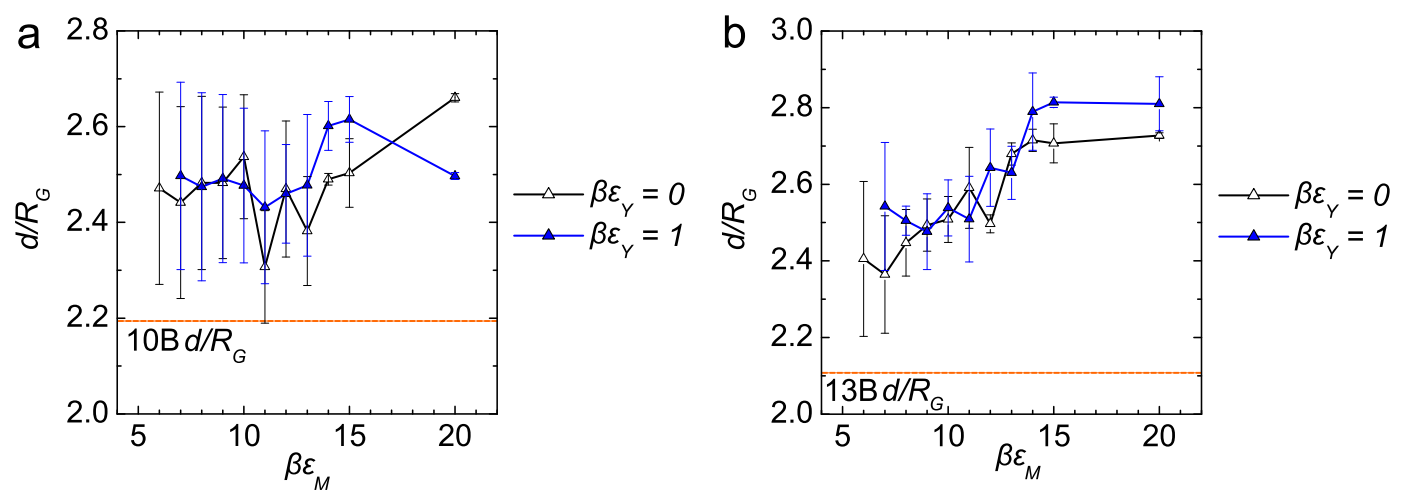

Figure 8. (color online) (a) Elongation $d / R_{G}$ for the $m=10$ (a) and $m=13$ (b) system as a function of the well depth of the attractive interaction $\beta \varepsilon_{M}$ with $\beta \varepsilon_{Y}=0$. The dashed orange line denotes the elongation in the case of the minimum energy ground state .

moderate values of $\beta \varepsilon_{M}$ it is not unreasonable to expect icosahedra. We expect that a similar kinetic argument to that suggested above for $m=10$ concerning the low yield of 10B holds for $m=13$ as well. For $m=10$ and $m=13$ we cannot exclude the possibility that other global minima exist. Doye et. al. [35] calculated global minima for $\rho_{0} \lesssim 25$. The smaller global energy minima for $m \leqslant 6$ exhibit no strain for short-ranged Morse interactions, and only a limited amount of strain in the case of $7 \mathrm{~A}$, these structures are therefore also expected to be minima for $\rho_{0}=33.06$ as used here. This not necessarily the case for $m=10$ and $m=13$. We are however unaware of any more appropriate structures than those listed in [35].

A question arises in comparing figure 7 with results for smaller clusters, such as the case of $m=3$ (figure 2). In general one expects that larger clusters should be able to form at higher temperatures (weaker interaction strengths) [1], (although for small Lennard-Jones clusters, the melting line is in fact non-monotonic as a function of $m$ [50]). Here, we are interested in whether all $m$ particles in the simulation box aggregate to form a cluster. This is less likely for larger $m$, so our statistics suffer for lower values of $\beta \varepsilon_{M}$ relative to the smaller clusters considered. It is nevertheless very clear that the yield of the assumed global minimum clusters is markedly reduced in the case of $m=10$ and $m=13$ relative to smaller clusters. We now proceed to consider elongation.

\subsection{Elongation}

The ground states of larger clusters have been found to be strongly affected by the strength of a Yukawa repulsion, with stronger repulsions leading to more elongated structures [36]. This effective long-ranged repulsion was indeed predicted to have a profound effect upon both the shape and the size of clusters of charged colloids by Groenewold and Kegel [42], and experiments have found evidence for elongated clusters [5, 7] and Bernal spirals [6]. We therefore investigate the degree of elongation of the larger clusters considered here. We consider the largest separation of two coordinates 
within a cluster, $d$, and divide this by the radius of gyration $R_{G}$ of the same coordinate set. Thus, larger $d / R_{G}$ corresponds to more elongated clusters, and for a large spherical cluster with $m \rightarrow \infty, d / R_{G} \rightarrow 2 \sqrt{5 / 3}$. For highly symmetric clusters such as icosahedra, $d / R_{G}=2.08$.

Figure 8 shows the elongation $d / R_{G}$ as a function of $\beta \varepsilon_{M}$. The elongation for $m=10$ and $m=13$ appears to be dominated by failure to access the ground state; nonergodic systems are more elongated. The data for $\beta \varepsilon_{Y}=1.0$ are included in figure 8, while for $\beta \varepsilon_{Y}=3.0$, few clusters formed at all, and none in the ergodic regime. From the discussion above, one might expect the $\beta \varepsilon_{Y}=1$ data to show a higher degree of elongation due to the Yukawa repulsion, however figure 8(a) indicates that this is not the case for $m=10$ and $m=13$, except for a slight hint at the lower values of $\beta \varepsilon_{M}$. We might expect that elongation induced by long-ranged repulsions is promoted by increasing $m$, or $\beta \varepsilon_{Y}$ or perhaps by a longer-ranged attraction that might suppress the transition to non-ergodicity, due to a less complex potential energy surface (see section 5) [51]. However in this system we see little evidence for elongation.

\section{Discussion}

The behaviour of the systems considered here can readily be decomposed into systems where frustration is not relevant, $m \leqslant 5$ where a well-defined structure is favoured, and those where frustration leads to a non-trivial potential energy landscape and limited access to the ground state $m \geqslant 7$. In the case of $m=6$ two structures with the same number of bonds, $6 \mathrm{~A}$ octahedra and $6 \mathrm{Z} D_{3 h}$ compete. Of particular relevance here is the short range of the attractive interactions. It has been noted previously that these tend to promote a complex energy landscape, for example $m=13$ Morse clusters with $\rho_{0}=14.0$ have some 54439 local energy minima, compared to just 685 for the longerranged case of $\rho_{0}=4.0$ [51]. The Morse potential with $\rho_{0}=14.0$ is an approximation to $C_{60}$, clusters of which are known to exhibit kinetic trapping [52, although in that case, icosahedral clusters were favoured. With $\rho_{0}=33.06$, stronger trapping is likely. What is clear, therefore, is that these systems can, only in the simplest cases $(m=3,4,5)$, form high yields of clusters in the minimum energy ground state, although $m=7$ does have a window in $\beta \varepsilon_{M}$ where the yield of $7 \mathrm{~A}$ is rather substantial.

\subsection{Relevance to experiments}

This work has been largely motivated by experimental studies of clustering in colloidal dispersions. In those systems, prized for their tunable interactions, the attractions, either Asakura-Oosawa, or van der Waals are typically rather short ranged, and thus likely to exhibit kinetic trapping similar to the systems studied here. We therefore argue that for substantial yields of more complex 'colloidal molecules', it is appropriate to seek more sophisticated means than the spherically symmetric spheres we have considered here, such as patchy particles [10, 14, 19, 41, 53] and Janus particles [16, 17], or to 
design routes of preparation [4, 54]. However, even 'purpose-designed' patchy particles can have only a rather limited window where the yield of ground state structures is substantial [11, 12, 13].

We now compare our results to those found in an experimental system in which

the interactions are, to a first approximation, identical [37]. Each of the simulations was conducted with a fixed number of of particles corresponding to the cluster size investigated. By contrast, most experiments are carried out with a bulk colloidal suspension, in which clusters of different size form. The experimental data report the relative abundance of cluster types for a fixed number of particles per cluster, $m$, and each cluster is assumed to interact only weakly with other clusters. In the experimental system, $m=3$ formed a majority of clusters in the $3 \mathrm{~A}$ triangle which maximises the number of bonds. This occurred at interaction strengths comparable to those found in the simulations reported here. In the experiments $m=4$ and 5 formed only around $10 \%$ of $4 \mathrm{~A}$ tetrahedra and $5 \mathrm{~A}$ triangular bipyramids respectively, in sharp contrast to the simulation results, where the yield was essentially $100 \%$. In the case of $m=6$, both in experiment and simulation, $6 \mathrm{Z} C_{2 v}$ dominated the $6 \mathrm{~A}$ octahedron. However, in the simulation, in the ergodic regime, the population difference was around a factor of 30, while in the experiment the difference was at least an order of magnitude larger. Furthermore, the maximum yield of $6 \mathrm{Z} C_{2 v}$ was an order of magnitude lower in the experiment than the simulation, where it was around $100 \%$. For $m=7$ both experiment and simulation show signs of geometric frustration, with the yield of $7 \mathrm{~A}$ being reduced in the nonergodic regime of strong interaction strength. However, while the peak yield of $7 \mathrm{~A}$ approached $100 \%$ in the simulation, the experiments were limited to a few percent of $7 \mathrm{~A}$. We consider possible origins for this discrepancy in the following section.

\subsection{Charging in apolar colloidal systems}

Before concluding, it is worth considering these results in the light of some other recent experimental studies. Campbell et. al. 6] report clusters in a system in which they measured the colloid charge in a dilute suspension to be $Z=140$ e per $1.5 \mu \mathrm{m}$ diameter colloid, where $e$ is the elementary charge. According to eq. (3), this maps to a Yukawa contact potential $\beta \varepsilon_{Y}=35$, their quoted value for the Debye length is comparable to ours. We have observed only very limited clustering at $\beta \varepsilon_{Y}=5$, corresponding to a charge of $Z=47 \mathrm{e}$ and expect none at higher Yukawa contact potentials. Dibble et. al. [8] quote a similar value of $Z=165$ e per $2 \mu \mathrm{m}$ colloid. Moreover Sedgwick et. al. [7] report $Z<10^{3} e$ in their study of clustering. Although not strictly inconsistent, this seems rather higher than the values we predict from eq. (3).

In their simulation study of gelation in colloidal systems with competing interactions, Sciortino et. al. [55] used a comparable contact potential for the Yukawa repulsion $\beta \varepsilon_{Y}$ to that used in the simulations here. In other words, a much weaker Yukawa repulsion than that expected from the colloid charge quoted by Campbell et. al. [6]. Interestingly, Sciortino et. al. [55] found that similar Bernal spiral 
structures were formed in their simulations to those observed experimentally [6]. From the arguments presented above, it might be supposed that the Bernal spiral can form without significant geometric frustration. An important question remains in the role of colloid concentration. We have considered a rather low volume fraction $\phi=m\left(\pi \sigma^{3} / 6\right) / l^{3}=0.0029$, as we are interested in isolated clusters. Whether the details of our results apply at finite concentration where clusters interact with one another remains to be seen, but many colloid volume fractions quoted in the literature are $\phi \lesssim 0.2$. In this regime we expect the discussion above regarding clustering in the presence of electrostatic repulsions to be reasonable.

Given the success of linear Poisson-Boltzmann theory in describing electrostatic interactions in these systems in the absence of polymer-induced attractions [56], one is tempted to enquire as to the origin of the discrepancy between the findings of this paper and the experimental literature [6, 7, 8]. As far as we know, in only one case has quantitative agreement been found between experiment and simulation of competing interactions using conventional models of electrostatic repulsion and polymer-induced attraction [57], and there the electrostatic repulsions were screened by salt. Combined with the discrepancies presented here and those in the experimental system [37] to which we have tuned the interactions, we believe that there is more than meets the eye to these colloidal model systems with competing interactions. We see little reason to suppose that the polymer-induced attractions deviate substantially from theory [44], therefore we speculate that the electrostatic repulsions in the clusters may deviate from those deduced by electrophoretic mobility measurements in dilute suspensions [6, 8, 37, 56].

\section{Conclusions}

We have studied isolated colloidal clusters using Brownian dynamics simulations. Our system is tuned to match experimental work in colloidal systems with polymer-induced depletion attractions. For sufficient strengths of the attractive interaction, the average bond lifetime exceeds the simulation runtime, and the system as nonergodic on this timescale; this conclusion also applies to some experimental systems. However, for small clusters $m \leqslant 5$ this ergodicity breaking does not prevent the system reaching the minimum energy ground state structure, as no bonds need be broken. Thus for these small clusters, we can direct the system in a controlled way towards a prescribed ground state. For $m=6$ we find a structure which appears to minimise the free energy with $C_{2 v}$ symmetry, rather than the octahedron that forms the minimum energy ground state [35, 36]. The energy difference between these structures is negligible, and for moderate interaction strengths (the ergodic regime) the $C_{2 v}$ structure is favoured for entropic reasons, while in the nonergodic regime, it is kinetically favoured as it is the product of an aggregation sequence that does not involve bond breakage. At larger cluster sizes, the system is kinetically frustrated from reaching the ground state in all but a limited window. Moreover, recent experimental results in a similar system show much lower yields of clusters in the ground state than we find here [37]. The origin of the 
discrepancy is likely related to a difference in the effective interaction potentials between experiment and simulation. It is perhaps surprising that such small systems exhibit this degree of kinetic trapping; only those sizes for which there is no geometric frustration $(m=3,4,5)$ reach the minimum energy ground state. These results suggest that high yields of complex microstructures of colloidal clusters and molecules may benefit from reversible quenching [38, 39, 40, 41], rather than the fixed interactions of many colloidal systems, which lead to 'instantaneous quenching'.

Some pointers for further work are considered. We have employed a simple approach in our simulations, as we are motivated to reproduce the recent experimental system. One approach to investigate larger clusters could be to run a simulation of a much larger number of particles, and to consider each cluster as a separate system. In this way, larger clusters could be accessed than was the case here. Although this could in principle resolve some discrepancies between the results presented here and those reported for the experimental system, we believe this is a most unlikely scenario. Other possibilities might be to implement the methods found in the (atomic) cluster literature [1, 35, 50] to comprehensively determine the structure of larger clusters. Another possibility would be to determine the phase diagram for the system considered here, using normal mode analysis to provide a theoretical prediction of the population levels for the various structures considered.

To provide further support to experimentalists in the quest to control the structure of colloidal clusters it might be helpful to move beyond the one-component description employed here. Given that the charging number quoted in some experimental work is

so small $(Z \lesssim 100 e)[6$, 7, [8, 37, [58], it might be possible to determine structures of colloidal clusters by for example developing the primitive model of electrolyte systems to colloidal systems with many ionisable sites on each particle [59].

\section{Acknowledgments}

This study was partly supported by EPSRC through grant code EP/E501214/1, and by a grant-in-aid from the Ministry of Education, Culture, Sports, Science and Technology, Japan. CPR acknowledges the Royal Society for financial support.

\section{References}

[1] F. Baletto and R. Ferrando. Structural properties of nanoclusters: Energetic, thermodynamic, and kinetic effects. Rev. Mod. Phys., 77:371-423, 2005.

[2] D. J. Wales. Energy Landscapes: Applications to Clusters, Biomolecules and Glasses. Cambridge University Press, Cambridge, Cambridge, 2004.

[3] P. N. Segre, V. Prasad, A. B. Schofield, and D. A. Weitz. Glasslike kinetic arrest at the colloidalgelation transition. Phys. Rev. Lett., 86:6042-6045, 2001.

[4] V. N. Manoharan, M. T. Elesser, and D. J. Pine. Dense packing and symmetry in small clusters of microspheres. Science, 301:483-487, 2003.

[5] A. Stradner, H. Sedgwick, F. Cardinaux, W.-C.K. Poon, S.U. Egelhaaf, and P. Schurtenberger. 
Equilibrium cluster formation in concentrated protein solutions and colloids. Nature, 432:492495, 2004.

[6] A. I. Campbell, V. J. Anderson, J. S. van Duijneveldt, and P. Bartlett. Dynamical arrest in attractive colloids: The effect of long-range repulsion. Phys. Rev. Lett., 94(20):208301, May 2005 .

[7] H. Sedgwick, S.U. Egelhaaf, and W.-C.K. Poon. Clusters and gels in systems of sticky particles. J. Phys:Condens. Matter, 16:S4913?S4922, 2004.

[8] C. J. Dibble, M. Kogan, and M. J. Solomon. Structure and dynamics of colloidal depletion gels: Coincidence of transitions and heterogeneity. Phys. Rev. E., 74:041403, 2006.

[9] J. N. Wilking, S. M. Graves, C. B. Change, K. Meleson, M. Y. Lin, and T. G. Mason. Dense cluster formation during aggregation and gelation of attractive slippery nanoemulsion droplets. Phys. Rev. Lett., 96:015501, 2006.

[10] S. C. Glotzer and M. J. Solomoin. Anisotropy of building blocks and their assembly into complex structures. Nature Materials, 6:557-562, 2007.

[11] A. W. Wilber, J. P. K. Doye, A. A. Louis, E. G. Noya, M. A. Miller, and P. Wong. Reversible self-assembly of patchy particles into monodisperse clusters. J. Chem. Phys., 127:085106, 2007.

[12] A. W. Wilber, J. P. K. Doye, A. A. Louis, and A. C. F. Lewis. Monodisperse self-assembly in a model with protein-like interactions. (http://arxiv.org/abs/0907.4811), arXiv:0907.4811v1 [cond-mat.soft], 2009.

[13] A. W. Wilber, J. P. K. Doye, and A. A. Louis. Topological classification of clusters in condensed phases. (http://arxiv.org/abs/0907.4807), arXiv:0907.4807v1 [cond-mat.soft], 2009.

[14] D. Zerrouki, J. Baudry, D. Pine, P. Chaikin, and J. Bibette. Chrial colloidal clusters. Nature, 455:380-382, 2008.

[15] S. M. Anthony, M. Kim, and S. Granick. Translation-rotation decoupling of colloidal clusters of various symmetries. J. CHem. Phys., 129:244701, 2008.

[16] L. Hong, A. Cacciuto, E. Luijten, and S. Granick. Clusters of charged Janus spheres. NanoLett., 6:2510-2514, 2006.

[17] L. Hong, A. Cacciuto, E. Luijten, and S. Granick. Clusters of amphiphillic colloidal spheres. Langmuir, 24:621-625, 2008.

[18] P. Akcora, H. Liu, S. K. Kumar, J. Moll, Y. Li, B. C. Benicewicz, D. Schadler, L. S. Acehan, A. Z. Panagiotopoulos, V. Pryamitsyn, V. Ganesan, J. Ilavsky, P. Thiyagarajan, and J. F. Colby, R. H. Douglas. Anisotropic self-assembly of spherical polymer-grafted nanoparticles. Natrue Mater., 8:354-359, 2009.

[19] Z. Zhang, A. S. Keys, T. Chen, and S. C. Glotzer. Self-assembly of patchy particles into diamond structures through molecular mimicry. Langmuir, 21:11547-11551, 2005.

[20] E. G. Noya, C. Vega, J. P. K. Doye, and A. A. Louis. Phase diagram of model anisotropic particles with octahedral symmetry. J. Phys. Chem., 127:054501, 2007.

[21] C.N. Likos. Effective interactions in soft condensed matter physics. Physics Reports, 348:267-439, 2001.

[22] H. Löwen and E. Allahyarov. The role of effective triplet interactions in charged colloidal suspensions. J. Phys.: Condens. Matter, 10:4147-4160, 1998.

[23] H. Kodama, K. Takeshita, T. Araki, and H. Tanaka. Fluid particle dynamics simulation of charged colloidal suspensions,. J. Phys.: Condens. Matter, 16:L115-L123, 2004.

[24] T. Araki and H. Tanaka. Physical principle for optimizing electrophoretic separation of charged particles,. Europhys. Lett., 82:18004, 2008.

[25] J.-P. Hansen and I.R. Macdonald. Theory of Simple Liquids. London: Academic press, 1976.

[26] D. Frenkel and B. Smit. Understanding Molecular Simulation: from Algorithms to Applications. New York: Academic, 2001.

[27] F. Sciortino, P. Tartaglia, and E. Zaccarelli. One-dimensional cluster growth and branching gels in colloidal systems with short-range depletion attraction and screened electrostatic repulsion. J. Phys. Chem. B., 109:21942-21953, 2005. 
[28] F. Sciortino, S. Mossa, E. Zaccarelli, and P. Tartaglia. Equilibrium cluster phases and low-density arrested disordered states: The role of short- range attraction and long-range repulsion. Phys. Rev. Lett., 93:055701, 2004.

[29] K. Kroy, M.E. Cates, and W.C.K. Poon. Cluster mode-coupling approach to weak gelation in attractive colloids. Phys. Rev. Lett., 92:148302, 2004.

[30] E. Zaccarelli. Colloidal gels: Equilibrium and non-equilibrium routes. J. Phys.: Condens. Matter, 19:323101, 2007.

[31] M. Tarzia and A. Coniglio. Pattern formation and glassy phase in the phi4 theory with a screened electrostatic repulsion. Phys. Rev. Lett., 96:075702, 2006.

[32] B. M. Mladek, D. Gottwald, G. Kahl, M. Neumann, and C. N. Likos. Formation of polymorphic cluster phases for a class of models of purely repulsive soft spheres. Phys. Rev. Lett., 96:045701, 2006.

[33] J. C. Fernandez Toledano, F. Sciortino, and E. Zaccarelli. Colloidal systems with competing interactions: from an arrested repulsive cluster phase to a gel. ArXiv:Cond Mat, 2009.

[34] Z.Y. Li, N.P. Young, M. Di Vece, S. Palomba, R.E. Palmer, A.L. Bleloch, B.C. Curley, R.L. Johnston, J. Jiang, and J. Yuan. Three-dimensional atomic-scale structure of size-selected gold nanoclusters. Nature, 451:46-49, 2008.

[35] J. P. K. Doye, D. J. Wales, and R. S. Berry. The effect of the range of the potential on the structures of clusters. J. Chem. Phys., 103(10):4234-4249, September 1995.

[36] S. Mossa, F. Sciortino, P. Tartaglia, and E. Zaccarelli. Ground-state clusters for short-range attractive and long-range repulsive potentials. Langmuir, 20:10756-10763, 2004.

[37] C. L. Klix, K. Murata, H. Tanaka, S. Williams, A. Malins, and C. P. Royall. The role of weak charging in metastable colloidal clusters. arXiv:0905.3393v1 [cond-mat.soft], (http://arxiv.org/abs/0905.3393), 2009.

[38] G. M. Whitesides and M. Boncheva. Beyond molecules: Self-assembly of mesoscopic and macroscopic components. Proc. Nat. Acad. Sci., 99:4769-4774, 2002.

[39] R. L. Jack, M. F. Hagan, and D. Chandler. Fluctuation-dissipation ratios in the dynamics of self-assembly. Phys. Rev. E., 76:021119, 2007.

[40] D. C. Rapaport. Role of reversibility in viral capsid growth: A paradigm for self-assembly. Phys. Rev. Lett., 101:186101, 2008.

[41] D. Nykypanchuk, M. M. Maye, D. van der Lelie, and O. Gang. DNA-guided crystallisation of colloidal nanoparticles. Nature, 451:549-552, 2008.

[42] J. Groenewold and W.K. Kegel. Anomalously large equilibrium clusters of colloids. J. Phys. Chem. B., 105:11702-11709, 2001.

[43] S. Asakura and F. Oosawa. On interaction between 2 bodies immersed in a solution of macromolecules. J. Chem. Phys., 22(7):1255-1256, 1954.

[44] C. P. Royall, A.A. Louis, and H. Tanaka. Measuring colloidal interactions with confocal microscopy. J. Chem. Phys., 127:044507, 2007.

[45] C. P. Royall, S. R. Williams, T. Ohtsuka, and H. Tanaka. Direct observation of a local structural mechanism for dynamic arrest. Nature Mater., 7:556-561, 2008.

[46] M. G. Noro and D. Frenkel. Extended corresponding-states behavior for particles with variable range attractions. J. Chem. Phys., 113:2941-2944, 2000.

[47] S. R. Williams. Topological classification of clusters in condensed phases. (http://arxiv.org/abs/0705.0203), arXiv:0705.0203v1 [cond-mat.soft], 2007.

[48] M. P. Allen and D. J. Tildesley. Computer Simulation of Liquids. Oxford University Press, Oxford, UK, 1989.

[49] J. P. K. Doye, D. J. Wales, and S. I. Simdyankin. Global optimization and the energy landscapes of Dzugutov clusters. Faraday Discuss., 118:159-170, 2001.

[50] J. P. K. Doye and F. Calvo. Entropic effects on the structure of Lennard-Jones clusters. J. Chem. Phys., 116:8307-8317, 2002.

[51] M. A. Miller, J. P. K. Doye, and D. J. Wales. Structural relaxation in Morse clusters: Energy 
landscapes. J. Chem. Phys., 110:328-334, 1999.

[52] F. Baletto, J. P. K. Doye, and R. Ferrando. Evidence of kinetic trapping in clusters of C60 molecules. Phys. Rev. Lett., 88:075503, 2002.

[53] . Park, S. Y, A. K. R. Lytton-Jean, B. Lee, S. Weigand, G. C. Schatz, and C. Mirkin. DNAprogrammable nanoparticle crystallisation. Nature, 451:553-556, 2008.

[54] K. E. Sung, S. A. Vanapalli, D. Mukhija, H. A. McKay, J. M. Millunchick, M. A. Burns, and M. J. Solomon. Programmable fluidic production of microparticles with configurable anisotropy. $J$. Am. Chem. Soc., 130:1335-1340, 2008.

[55] F. Sciortino and P. Tartaglia. Glassy colloidal systems. Adv. Phys., 54(6-7):471-524, 2005.

[56] C.P. Royall, M.E. Leunissen, A.-P. Hynninen, M. Dijkstra, and A. van Blaaderen. Re-entrant melting and freezing in a model system of charged colloids. J. Chem. Phys., 124:244706, 2006.

[57] C.P. Royall, D.G.A.L. Aarts, and H. Tanaka. Fluid structure in colloid-polymer mixtures: the competition between electrostatics and depletion. J. Phys:Condens. Matter, 17:S3401-S3408, 2005 .

[58] G.S. Roberts, T.A. Wood, W.J. Frith, and P. Bartlett. Direct measurement of the effective charge in nonpolar suspensions by optical tracking of single particles. Cond-mat, 2007.

[59] V. Lobaskin and P. Linse. Simulation of an asymmetric electrolyte with charge asymmetry 60:1 using hard-sphere and soft-sphere models. J. Chem. Phys., 111:4300-4309, 1999. 